ARTICLE

\title{
Toward a generalized Bienenstock-Cooper-Munro rule for spatiotemporal learning via triplet-STDP in memristive devices
}

\author{
Zhongqiang Wang ${ }^{1,3}$, Tao Zeng ${ }^{1,3}$, Yanyun Ren ${ }^{1}$, Ya Lin ${ }^{1}$, Haiyang $X u^{1 凶}$, Xiaoning Zhao ${ }^{1}$, Yichun Liu ${ }^{1 凶} \&$ \\ Daniele lelmini (iD) $2 \times$
}

The close replication of synaptic functions is an important objective for achieving a highly realistic memristor-based cognitive computation. The emulation of neurobiological learning rules may allow the development of neuromorphic systems that continuously learn without supervision. In this work, the Bienenstock-Cooper-Munro learning rule, as a typical case of spike-rate-dependent plasticity, is mimicked using a generalized triplet-spike-timingdependent plasticity scheme in a $\mathrm{WO}_{3-x}$ memristive synapse. It demonstrates both presynaptic and postsynaptic activities and remedies the absence of the enhanced depression effect in the depression region, allowing a better description of the biological counterpart. The threshold sliding effect of Bienenstock-Cooper-Munro rule is realized using a historydependent property of the second-order memristor. Rate-based orientation selectivity is demonstrated in a simulated feedforward memristive network with this generalized Bienenstock-Cooper-Munro framework. These findings provide a feasible approach for mimicking Bienenstock-Cooper-Munro learning rules in memristors, and support the applications of spatiotemporal coding and learning using memristive networks.

\footnotetext{
${ }^{1}$ Key Laboratory for UV Light-Emitting Materials and Technology (Northeast Normal University), Ministry of Education, Renmin Street, 5268 Changchun, China. ${ }^{2}$ Dipartimento di Elettronica, Informazione e Bioingegneria, Politecnico di Milano, Piazza L. da Vinci 32, 20133 Milano, Italy. ${ }^{3}$ These authors contributed equally: Zhongqiang Wang, Tao Zeng. ${ }^{凶}$ email: hyxu@nenu.edu.cn; ycliu@nenu.edu.cn; daniele.ielmini@polimi.it
} 
B rain-inspired neuromorphic computing systems are attracting strong interest because of their massive parallelism, high energy efficiency, good error tolerance, and good ability to implement cognitive functions ${ }^{1-6}$. Hardware implementations of neuromorphic computing can take advantage of novel nanodevices that emulate the biological synapses with inherent learning functions ${ }^{7-13}$. The two-terminal memristor is widely recognized as a promising technology with which to mimic the biological synapse because of its functional resemblance to the biological counterpart ${ }^{14-19}$. The biorealistic realization of synaptic plasticity in the memristor is considered to be an important step toward realizing an artificial synapse with high accuracy. There have been many efforts to demonstrate basic synaptic learning functions using single and paired spikes, for example, long-term/ short-term plasticity, spike-timing-dependent plasticity (STDP), and paired-pulse facilitation $(\mathrm{PPF}) /$ depression $^{20-27}$. In fact, the stimulation mode of a spike train that contains plentiful spikes is a more general case than the single spike or paired spikes, and is produced by a neuron receiving multiple spikes from other connected neurons ${ }^{28}$. The information contained in a spike train allows specific advanced plasticity within a synapse that is referred to as spike-rate-dependent plasticity (SRDP) ${ }^{29}$.

The Bienenstock-Cooper-Munro (BCM) learning rule is an important type of SRDP beyond the Hebbian learning rule and describes history-dependent synaptic modification. In the BCM framework, the high/low spike rate of a train can result in the potentiation/depression of the synaptic weight depending on whether the spike rate is higher than a threshold $(\theta)^{30-33}$. For the memristor-based artificial synapse, several groups have demonstrated BCM rules using rate-based presynaptic spikes, which have led to advances in the field ${ }^{34-36}$. These results show that the absolute change in the synaptic weight (i.e., the conductance change of the memristor, $\left.\left|\Delta G_{c}\right|\right)$ has a monotonic dependence on the spike rates in both the depression region $\left(\Delta G_{c}<0\right)$ and potentiation region $\left(\Delta G_{c}>0\right)$. However, such a monotonic change is different from the original BCM rule in neurobiology; that is, there should exist non-monotonic behavior (i.e., an enhanced depression effect $(\mathrm{EDE}))$ in the depression region ${ }^{30-33,37,38}$. Additionally, previous memristor studies lack the following essential features: first, the lack of a multiplicative term between presynaptic and postsynaptic activities, and second the short-term modification ${ }^{34-36}$. This also marks a significant inconsistency with the biological BCM learning.

According to a theoretical model of Pfister et al., it is expected that the use of triplet-STDP, instead of common rate-based presynaptic spikes, allows this issue to be solved ${ }^{39,40}$. Furthermore, the BCM rule can be generalized by the long-term triplet-STDP, thereby allowing higher-order spatiotemporal recognition in the visual cortex, for example, rate-based orientation selectivity ${ }^{39}$. Triplet-STDP means that a third spike, either presynaptic or postsynaptic, is introduced into the standard pair-STDP $33,40-42$. Importantly, in addition to the paired term contribution in pair spikes, a previous spike (presynaptic or postsynaptic) also causes the contribution of a triplet term in the triplet-STDP $33,41,42$. The relationship between the paired term and triplet term contributions provides the multiplicative correlations between presynaptic and postsynaptic activities, which is an essential requisite for BCM learning. There are two types of triplet-STDP in neuroscience: the first-spike-dominating model and last-spike-dominating model proposed by Froemke et al. and Wang et al., respectively ${ }^{41,42}$. Progress has been made in emulating these two types of tripletSTDP using first-order and second-order memristors ${ }^{36,43-45}$. However, the generalization from triplet-STDP to the BCM learning rule has not yet been experimentally demonstrated in memristors. Additionally, high-order spatiotemporal recognition that relies on generalized BCM learning rules has rarely been reported.
The present work presents the demonstration of generalized $\mathrm{BCM}$ learning rules using the last-spike-dominating triplet-STDP in a $\mathrm{WO}_{3-\mathrm{x}}$-based second-order memristor. The second-order memristor has physical behavior similar to $\mathrm{Ca}^{2+}$ dynamics in the bioneural network, which allows the emulation of rate-based plasticity naturally $16,34,46$. The EDE, which was typically missing in previous studies, is achieved using a long-term triplet-STDP scheme. Our experimental results are highly consistent with the mathematical model of the BCM framework in a biological system. Additionally, rate-based orientation selectivity is demonstrated on the basis of such a generalized triplet-STDP-based BCM learning rule, showing its strong potential in high-order spatiotemporal recognition.

\section{Results}

Motivation and $\mathrm{WO}_{3-\mathrm{x}}$ second-order memristor. Figure 1a depicts the motivation for a biologically plausible triplet-STDPbased BCM learning rule in memristive hardware. Generally, neurons in a neurophysiological system interact by exchanging spike trains, that is, sequences of pre- and postsynaptic spikes. Because of the diversity of the spike trains, the synaptic modification is not only determined by the timing between paired spike, as in the case of common STDP, but also affected by the details of the spike pattern, such as the spike train rate $23,29,47$. A relatively simple model of pattern-dependent plasticity is tripletSTDP $^{41,42}$. Typical BCM learning rules can then be generalized based on triplet-STDP, which also allows rate-based orientation selectivity for high-order spatiotemporal functions.

To demonstrate the aforementioned synaptic functions, we considered the $\mathrm{WO}_{3-\mathrm{x}}$-based second-order memristors illustrated in Fig. 1b, which consist of a $\mathrm{Pt} / \mathrm{WO}_{3-\mathrm{x}} / \mathrm{W}$ sandwich structure prepared in crossbar arrays using a sputtering deposition technique. Figure $1 \mathrm{c}$ shows the $\mathrm{I}-\mathrm{V}$ curve of the $\mathrm{WO}_{3-\mathrm{x}}$-based memristor, gradually increases and decreases with positive- and negative-bias sweeps on the top Pt electrode. The conductance $G_{c}$ can be regarded as the synaptic weight. The asymmetric I-V curve of Fig. 1c also indicates the existence of Schottky barrier at Pt/ $\mathrm{WO}_{3-\mathrm{x}}$ interface. This memristive behavior is similar to the nonlinear transmission of a biological synapse, thereby allowing for a continuous adjustment of synaptic weight. Analogous to biological spikes, a series of positive and negative pulses elicits the consecutive potentiation and depression of our memristive synapse, as shown in Fig. 1d. Additionally, the potentiation state can spontaneously decay to a middle state, as shown in Fig. 1e, which is a clear indicator of the second-order memristor (Supplementary Fig. 1 and Note 1) ${ }^{16,17,34}$. Such behavior is equivalent to a transition from short-term plasticity to long-term plasticity by repeated stimulation ${ }^{21}$. The memristor dynamics are caused by oxygen-ion diffusion that resembles biological $\mathrm{Ca}^{2+}$ dynamics ${ }^{15,16,21,24}$. Du et al. previously developed a $\mathrm{WO}_{\mathrm{x}}$ memristive device with a $\mathrm{Pd} / \mathrm{WO}_{\mathrm{x}} / \mathrm{W}$ structure where the switching mechanism was attributed to the modification of a relative area of the conducting channel ${ }^{34}$. In our work, instead, the Schottky barrier of the $\mathrm{Pt} / \mathrm{WO}_{3-\mathrm{x}}$ interface and its modulation induced by the drift and diffusion of oxygen ions can account for the memristive mechanism (Supplementary Fig. 1 and Note 1). The different device fabrication methods and electrodes may be responsible for the different memristive behaviors. A similar Schottky barrier based memristive mechanism was reported in our previous work and other literature ${ }^{22,36,44,48}$.

Synaptic adaptation function emulated by rate-based postsynaptic spikes. The synaptic adaptation function was mimicked using a second-order memristive effect in the $\mathrm{WO}_{3-\mathrm{x}}$ device. Figure $2 \mathrm{a}$ shows the current response of the memristive device 
a

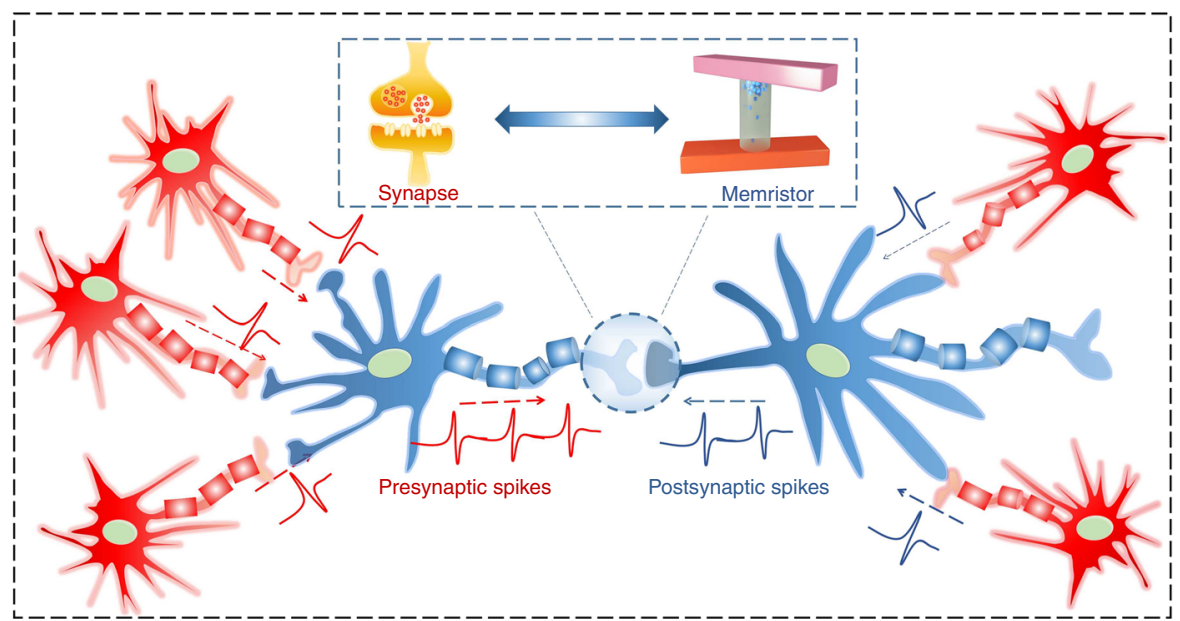

b
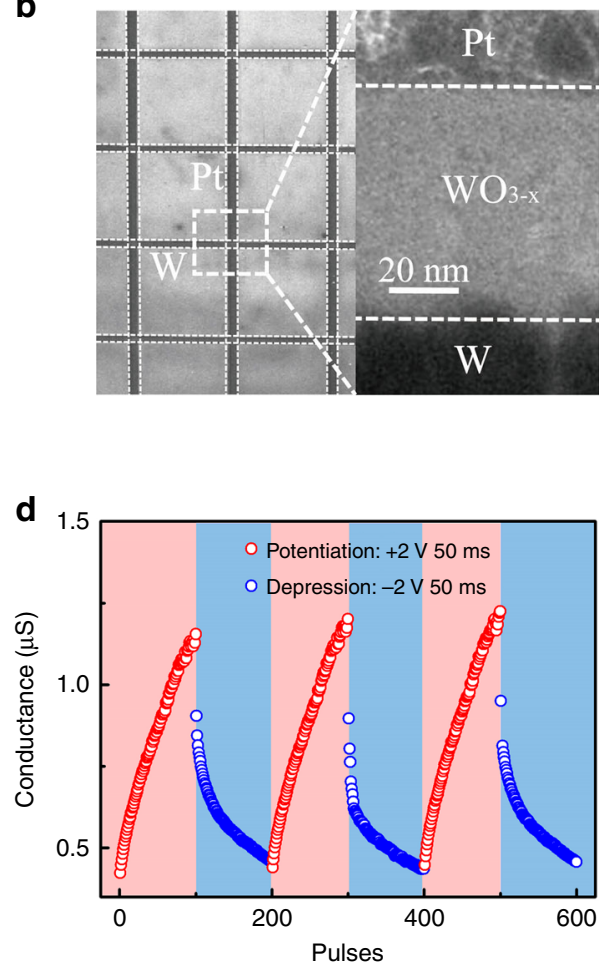

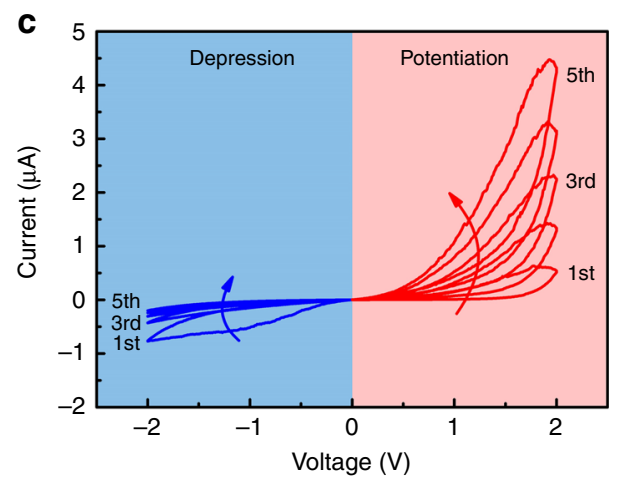

e

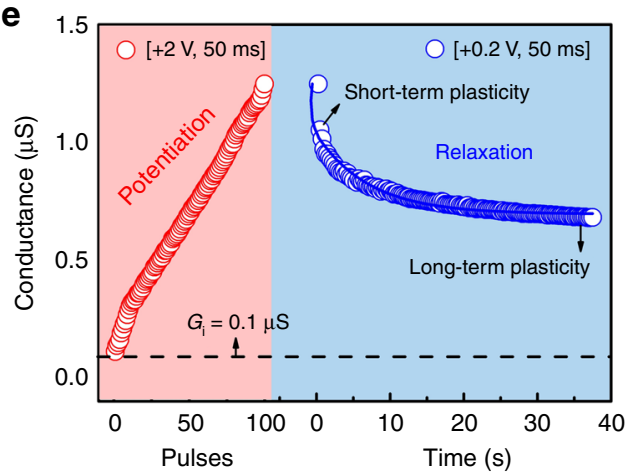

Fig. 1 Artificial synapse based on the $\mathbf{W O}_{3-\mathbf{x}}$ second-order memristor. a Schematic of spike trains in a biologic system, produced by a neuron receiving multiple spikes from other connected neurons. b Scanning electron microscopy image of the memristor crossbar array and a transmission electron microscopy image of the cross-section of the Pt/ $\mathrm{WO}_{3-x} / \mathrm{W}$ memristor. $\mathbf{c} \mathrm{I}-\mathrm{V}$ curve of the memristor under a continuous positive sweep ( 0 to $2 \mathrm{~V}$ ) and negative sweep $\left(0\right.$ to $-2 \mathrm{~V}$ ). d Potentiation and depression of conductance $G_{c}$ caused by the repeated stimulation of 100 positive pulses $[+2 \mathrm{~V}$, 50 ms $]$ and 100 negative pulses $[-2 \mathrm{~V}, 50 \mathrm{~ms}]$. All the data of $G_{c}$ were collected $1 \mathrm{~s}$ after the stimulation using a reading pulse $[+0.2 \mathrm{~V}, 50 \mathrm{~ms}]$, which represents the long-term plasticity. e Spontaneous decay of conductance $G_{c}$ after the potentiation process. This decay process may be related to the diffusion of oxygen ions, which is an indicator of a second-order memristor. Here, the device was operated from an initial conductance $G_{i}$ of $0.1 \mu S$. The relaxation process was monitored under a read pulse $[+0.2 \mathrm{~V}, 50 \mathrm{~ms}]$.

under a single presynaptic spike $[2 \mathrm{~V}, 10 \mathrm{~ms}]$. The figure shows that the presynaptic spike triggers an abrupt increase in current followed by a decay to the initial state within $400 \mathrm{~ms}$, which is similar to the behavior of the excitatory postsynaptic current (EPSC) of the biological synapse ${ }^{21,24}$. The similarity with the biological EPSC behavior is caused by oxygen-ion drift and diffusion in our memristor paralleling the influx and extrusion of $\mathrm{Ca}^{2+}$ through the synaptic cell ${ }^{24,49}$. It is noted in Fig. 2a that there is not an abrupt decrease in current observed, when the stimulation pulse of $2 \mathrm{~V}$ is changed to the monitoring pulse of 0.2 $\mathrm{V}$. One of the possible reasons is the electric double-layer (EDL) capacitance, which is discussed in a recent paper by Yang et al. ${ }^{50}$. In a biological system, the $\mathrm{Ca}^{2+}$ dynamics allow correlation between paired spikes, where the residual $\mathrm{Ca}^{2+}$ induced by the first spike enhances the overall $\mathrm{Ca}^{2+}$ concentration generated by the second spike, thus resulting in PPF. The PPF function is critical for a synapse to make correlations between the temporal spike pair. Based on the similarity between oxygen-ion dynamics and $\mathrm{Ca}^{2+}$ dynamics, we can demonstrate PPF, as shown in Fig. $2 \mathrm{~b}$. When the second stimulation comes before the first EPSC disappears completely, their overlap can effectively suppress the diffusion of oxygen ions. This promotes the more effective 

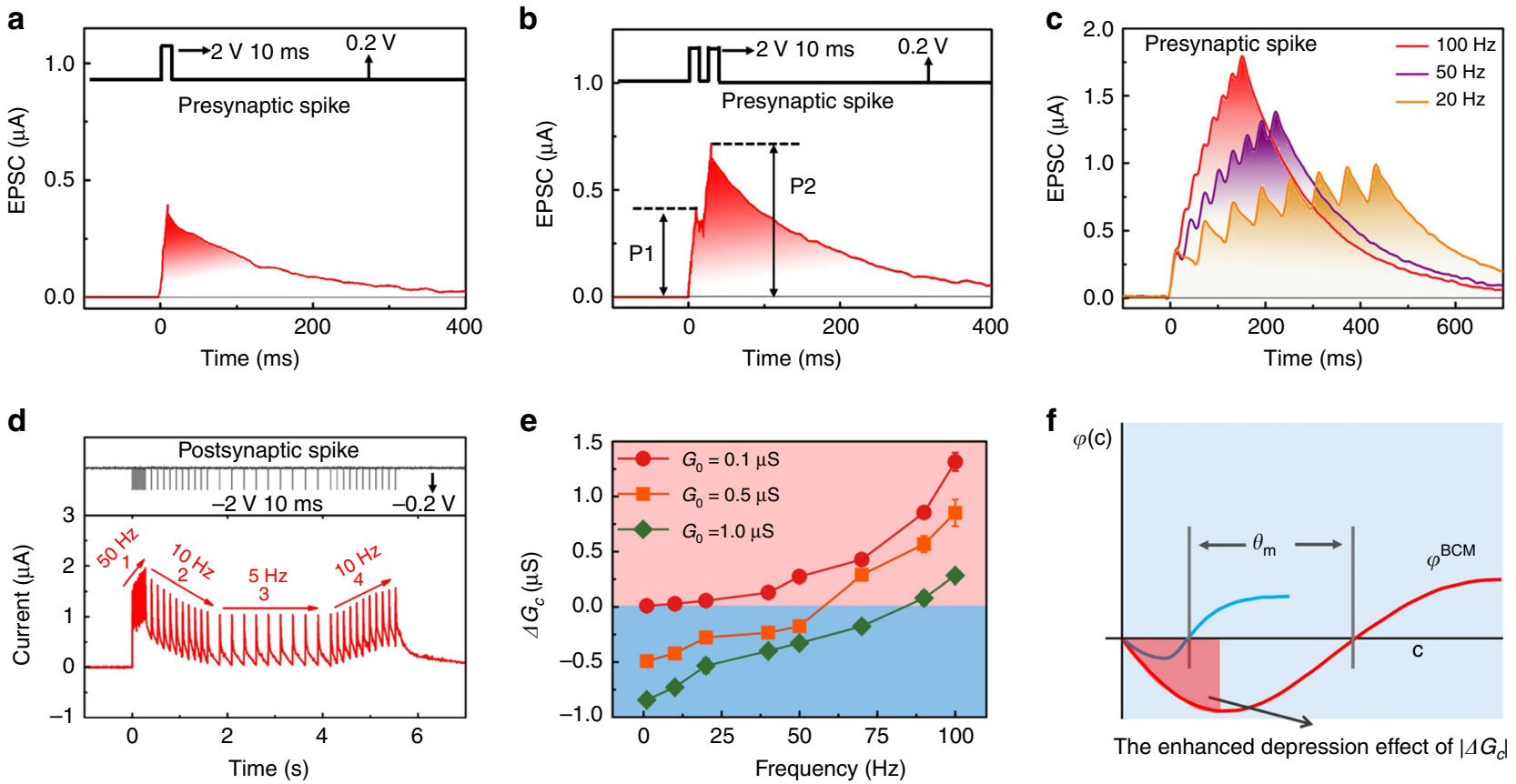

Fig. 2 Emulation of the rate-based synaptic adaptation function using postsynaptic spikes in the $\mathbf{P t} / \mathbf{W O}_{\mathbf{3}-\mathbf{x}} / \mathbf{W}$ memristors. a, $\mathbf{b}$ Synaptic EPSC and PPF functions triggered by a presynaptic spike $[2 \mathrm{~V}, 10 \mathrm{~ms}]$ and paired spikes. c Dependence of the EPSC amplitude on the rate of presynaptic spike trains (20, 50 , and $100 \mathrm{~Hz}$ ). Eight pulses $[2 \mathrm{~V}, 10 \mathrm{~ms}]$ were used to stimulate the device. d Response of the synaptic weight (conductance $G_{c}$ ) to a group of postsynaptic spike trains with a frequency sequence $(50 \mathrm{~Hz} \rightarrow 10 \mathrm{~Hz} \rightarrow 5 \mathrm{~Hz} \rightarrow 10 \mathrm{~Hz})$. The pulse [-2V, $10 \mathrm{~ms}$ ] on the bottom electrode as the postsynaptic spike. e Sliding threshold effect of the history-dependent synaptic adaptation function with different $G_{0}$. Herein, the experienced $G_{0}$ was activated to three levels starting from a fixed initial conductance of $G_{i}=0.1 \mu \mathrm{S}$. For the case of $G_{0}=0.5 \mu S$ and $1.0 \mu \mathrm{S}$, the stimulation was conducted using a pulse amplitude of $-2 \mathrm{~V}$ and pulse width of $10 \mathrm{~ms}$ with postsynaptic spike rates of $20 \mathrm{~Hz}$ and $50 \mathrm{~Hz}$, respectively. The peak of temporary conductance potentiation $\left(G_{\text {peak }}\right.$ ) was collected to calculate $\Delta G_{c}=G_{\text {peak }}-G_{0}$, which represents a type of short-term plasticity. $\mathbf{f}$ Biological $B C M$ curve. The vertical and horizontal axes are the weight modification $\varphi(c)$ and postsynaptic firing rate $c$, respectively. According to the $B C M$ function of $\varphi^{B C M}$, the depression/ potentiation of $\varphi(c)$ occurs as the firing rate is lower/higher than modification threshold $\theta_{m}$. In particular, the parameter $\theta_{m}$ is adaptive to the experienced activity: the synapse which experienced a period of inactivity would follow the blue curve with smaller $\theta_{m}$, whereas the synapse that experienced a period of enhanced activity would follow the red curve. Reproduced with permission ${ }^{32}$. Copyright 2012, Nature Pub. Group. The red shaded area indicates an EDE of I $\Delta G_{c}$ in the depression region, which was usually absent in previous memristor-based BCM studies.

accumulation of oxygen ions on the $\mathrm{Pt} / \mathrm{WO}_{3-\mathrm{x}}$ interface, which leads to a larger conductance change. Thus, the peak value of EPSC induced by the second spike P2 is clearly higher than that of the first-spike P1, and a longer interval between presynaptic spikes gradually decreases the facilitation of the second spike (Supplementary Fig. 2). In fact, the EPSC evoked by a single spike results in a temporary effect (i.e., short-term plasticity), whereas the PPF with sufficiently large spikes results in a permanent effect (i.e., conversion from short-term plasticity to long-term plasticity). The PPF effect can also be extended to the SRDP using spike trains instead of spike pairs, as shown in Fig. 2c. In this case, the increase in EPSC amplitude depends on not only the presynaptic spike number but also the spike rate. Spikes with a higher rate (i.e., a shorter interval) result in a much larger EPSC amplitude, similar to the case of SRDP in the biological synapse ${ }^{51-53}$.

History-dependent plasticity is an important behavior for the synaptic adaptation function. To verify the feasibility of historydependent plasticity for our memristor, we applied a pulse $[-2 \mathrm{~V}$, $10 \mathrm{~ms}$ ] to the bottom electrode as the postsynaptic spike in Fig. 2d, including four distinct phases. In the first phase, a postsynaptic spike train at a relatively high frequency $(50 \mathrm{~Hz})$ increased the conductance in the memristor. In the second phase, the lower frequency $(10 \mathrm{~Hz})$ of the spike train decreased the conductance (i.e., synaptic depression). A spike train of $5 \mathrm{~Hz}$ in the third phase and a final spike train of $10 \mathrm{~Hz}$ applied in the fourth phase increased the conductance again (i.e., synaptic potentiation). It is interesting to note that the second and fourth phases induced opposing conductance changes even though the same spike trains of $10 \mathrm{~Hz}$ were used. In this experiment, the conductance state after stimulation at 50 and $5 \mathrm{~Hz}$ can be regarded as the experienced conductance $\left(G_{0}\right)$; that is, different 'experiences' or 'histories.' The postsynaptic spike train of $10 \mathrm{~Hz}$ can thus induce depression or potentiation depending on the experienced $G_{0}$ activated by higher $(50 \mathrm{~Hz})$ or lower $(5 \mathrm{~Hz})$ frequencies, thereby indicating the realization of historydependent plasticity.

The postsynaptic spike-rate-based synaptic adaptation function was further implemented in $\mathrm{WO}_{3-\mathrm{x}}$ memristors by changing the learning experience, hence $G_{0}$, as illustrated in Fig. 2e. Spiking at relatively low and high frequencies generally results in depression and potentiation, respectively, in line with the results in Fig. $2 \mathrm{~d}$. Additionally, the value of $G_{0}$ dictates the threshold rate $\theta$, which shows a formalistic similarity with the sliding threshold effect of the $\mathrm{BCM}$ rule $\mathrm{in}^{30-33}$. For this reason, previous studies demonstrated the BCM learning by the implementation of results similar to Fig. $2 \mathrm{e}^{34-36}$. Strictly speaking, however, such synaptic adaptation function is not fully equivalent to the biological BCM rule, given three fundamental differences between them: (i) The observed synaptic adaptation genrally consists of a temporary short-term modification of the synaptic weight, while BCM learning should refer to long-term potentiation/depression (LTP/ LTD) according to the literature ${ }^{30-33}$. (ii) In BCM learning, the synaptic weight is a function of both the presynaptic and 
postsynaptic neuron activities and also requires a multiplicative relationship between them. On the other hand, only postsynaptic spikes were used in the synaptic adaptation function implemented in our work and previous studies ${ }^{34-36}$. (iii) Note that conductance change $\Delta G_{c}$ is a monotonic function of the spike rate in both the depression and potentiation regions, which is obviously different from the biological BCM model of Fig. $2 \mathrm{f}^{32}$. In the depression region, the depression effect should first enhance (i.e., $\left|\Delta G_{c}\right|$ increases) at a relatively low spike rate and then weaken as the spike rate increases; that is, the EDE in the depression region (the red shaded area in Fig. 2f) was missing in the existing implementation of the BCM rule using rate-based postsynaptic spikes (Fig. 2e) ) $^{34-36}$. The absence of the EDE can be understood in that there are only two competitive factors that determine synaptic change $\Delta G_{c}$ : the spontaneous forgetting effect of the experienced $G_{0}$ and the potentiation effect induced by the presynaptic spike train ${ }^{34-36}$. There is no other rate-based depression effect that can assist the forgetting effect to induce the EDE. It is essential to remedy this EDE region in memristors to closely approximate the biological synapse; however, there is a lack of related studies.

Triplet-STDP and generalized BCM learning rule. Different from previous implementations using common rate-based postsynaptic spikes, in theory, the BCM learning rule can be closely replicated using a generalized model based on triplet-STDP 39,40 . This generalized triplet-STDP-based BCM rule is experimentally demonstrated in our memristive devices. The long-term pairedSTDP was first implemented on an experimental basis and as a comparison for triplet-STDP. For the paired-STDP, the time delay between paired spikes (i.e., $\Delta t=t_{\text {post }}-t_{\text {pre }}$ ) determines whether the LTP at $\Delta t>0$ (i.e., the presynaptic spike is earlier than the postsynaptic spike) or LTD at $\Delta t<0$ occurs, which was also demonstrated in our $\mathrm{WO}_{3-\mathrm{x}}$-based memristors (Supplementary Fig. 3 and Note 2). For the long-term triplet-STDP, the spike train can be assumed to be the combination of two spike pairing events, and the synaptic modification can thus be regarded as the integration of LTP and LTD processes induced by these two events ${ }^{42}$. Additionally, the triplet term induced by the previous spike to the paired spikes also needs to be taken into account. Figure $3 \mathrm{a}$ illustrates two typical triplets with 'post-prepost' and 'pre-post-pre' sequences. For the 'post-pre-post' triplet, the LTD process is induced by the first-spike pairing ('post-pre', $\left.\Delta t_{1}<0\right)$, which is followed by an LTP process induced by the second spike pairing ('pre-post', $\Delta t_{2}>0$ ). For the 'pre-post-pre' triplet, the LTP process is activated before the LTD process. Hereafter, the pair $\left(\Delta t_{1}, \Delta t_{2}\right)$ is used to denote the spike timing in triplets. This means that the 'post-pre-post' triplet always has the timing of $\Delta t_{1}<0$ and $\Delta t_{2}>0$, whereas the 'pre-post-pre' triplet has the timing of $\Delta t_{1}>0$ and $\Delta t_{2}<0$. In the triplet-STDP scheme, each spike applied on the memristor consists of a pair of pulses $\left[V^{+} / V^{-}=2 \mathrm{~V} /-2 \mathrm{~V}, 50 \mathrm{~ms}\right]$ as illustrated in Fig. 3a and in our previous work ${ }^{21,22}$. Refer to Supplementary Fig. 3 for details of the spike design; a delay time of $60 \mathrm{~s}$ was introduced to ensure the readout of long-term conductance change before and after the spikes ${ }^{21}$.

The synaptic modification of triplet-STDP was examined with the symmetrical spike timing of the LTP and LTD processes (i.e., $\left|\Delta t_{1}\right|=\left|\Delta t_{2}\right|$ ), as shown in Fig. 3b. For the 'post-pre-post' triplet, the obvious potentiation of $\Delta G_{c}$ was observed in the case of $G_{0}=0.1 \mu \mathrm{S}$ (see the red circle in Fig. 3b), whereas the potentiation exponentially decreased with increasing spike timing $\Delta t$. For the 'pre-post-pre' triplet, there was no notable change in $\Delta G_{c}$ after the stimulation in the case of $G_{0}=0.1 \mu \mathrm{S}$, which indicates that the LTP process was canceled by the following LTD process. In fact, the asymmetrical result of these two triplet sequences cannot be understood by simply considering the competition of LTP and LTD in the paired-STDP model ${ }^{39,40}$. In that interpretation, the LTP and LTD processes should counteract each other in both triplets regardless of their sequence. Interestingly, this result presented a demonstration of the last-spike-dominating triplet-STDP, and a similar finding was reported in a neurobiological study published by Wang et al. ${ }^{42}$. Furthermore, the effect of the long-term learning experience (i.e., different $G_{0}$ measured in long-term plasticity) on triplet-STDP can be observed in Fig. 3b. Our previous work indicates that the stimulation from an intermediate state with long-term learning experience can induce a larger $\Delta G_{c}{ }^{21}$. The physical mechanism may be related to the metastable local structure (e.g., unstable interstitial oxygen ions) with a lower energy barrier for further defect migration ${ }^{21}$. For the case $G_{0}=0.5 \mu \mathrm{S}$, the potentiation was reduced overall, even to the extent that the depression appeared for a relatively long spike timing in the 'post-pre-post' triplet, whereas the depression strengthened for higher $G_{0}$ in the 'prepost-pre' triplet. In particular, when $G_{0}$ increased to $3.0 \mu \mathrm{S}, \Delta G_{c}$ in the 'post-pre-post' triplet was no longer a monotonic function of the spike interval $\left(\Delta t_{1}=\Delta t_{2}\right)$. The potentiation of $\Delta G_{c}$ happened for a relatively short interval time $(\Delta t<8 \mathrm{~ms})$, while a longer interval time induced the depression of $\Delta G_{c}$, as illustrated by the green-diamond data in Fig. $3 \mathrm{~b}$. This behavior is similar to the biological BCM learning rule when converting the interval time into the frequency ${ }^{39,40}$. This provides an experimental foundation with which to remedy the absence of the EDE region in the BCM learning rule, which will be demonstrated later. As discussed previously, there are only two competitive factors in determining $\Delta G_{c}$ in the BCM rule implemented using common rate-based postsynaptic spikes, which leads to the absence of the EDE region. By contrast, an additional depression effect of the LTD process is introduced in triplet-STDP. Together with the forgetting effect of experienced $G_{0}$ and the potentiation effect of the LTP process, there is a third competitive factor in triplet-STDP. With the help of the forgetting effect of high $G_{0}$, this third factor, that is, the EDE of the LTD process (Supplementary Fig. 4), could make up for the absence of the EDE region, as illustrated in Fig. 2f. Meanwhile, the asymmetrical spike-timing intervals played a critical role in the competition of LTP and LTD processes, as shown in Fig. 3c. In the 'post-pre-post' case, $\Delta G_{c}$ transformed from potentiation to depression as the interval of the LTP process $\left(\Delta t_{2}\right)$ increased from 10 to $120 \mathrm{~ms}$ whereas the interval of the LTD process $\left(\Delta t_{1}=-70 \mathrm{~ms}\right)$ was kept constant. Similarly, in the 'prepost-pre' case, the depression of $\Delta G_{c}$ weakened with increasing $\left|\Delta t_{2}\right|$ and while keeping $\Delta t_{1}=70 \mathrm{~ms}$ constant.

Figure $3 \mathrm{~d}$, e summarize the data of the last-spike-dominating triplet-STDP with varying $\Delta \mathrm{t}_{1}$ and $\Delta \mathrm{t}_{2}$ using a colored background to show $\Delta G_{c}$. In addition to the mentioned 'post-pre-post' and 'pre-post-pre' cases, four other patterns (i.e., two pre-spikes (postspikes) occur before and after a single post-spike (pre-spike)) were included, as shown in quadrants I and III of Fig. 3d, e. Generally, the potentiation effect was observed in quadrant I because the prespike was always applied before the post-spike regardless of the spike number, similar to the LTP process of paired-STDP. By contrast, the depression effect was dominant in quadrant III, similar to the LTD process of paired-STDP. For quadrants II and IV, the synaptic weight $G_{c}$ can be switched from depression to potentiation by adjusting the timing of $\Delta t_{1}$ and $\Delta t_{2}$, which is in accordance with the results of Fig. $3 \mathrm{~b}$, c. The results shown in Fig. 3d, e also indicate the possible dependence of $\Delta G_{c}$ on the interval time between two postsynaptic spikes $\Delta t_{o}=t_{\text {post }}-t_{\text {post }}$ and that between two presynaptic spikes $\Delta t_{r}=t_{\text {pre }}^{\prime}-t_{\text {pre, }}$, where $t_{\text {post }}, t_{\text {post }}^{\prime}, t_{\text {pre, }}$ and $t_{\text {pre }}^{\prime}$ are the times of the two postsynaptic spikes and two presynaptic spikes. In fact, the dependence of $\Delta G_{c}$ 
a

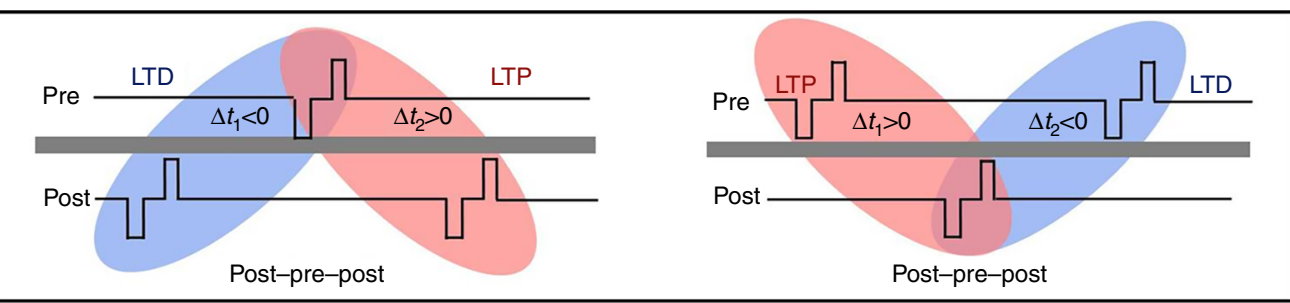

b

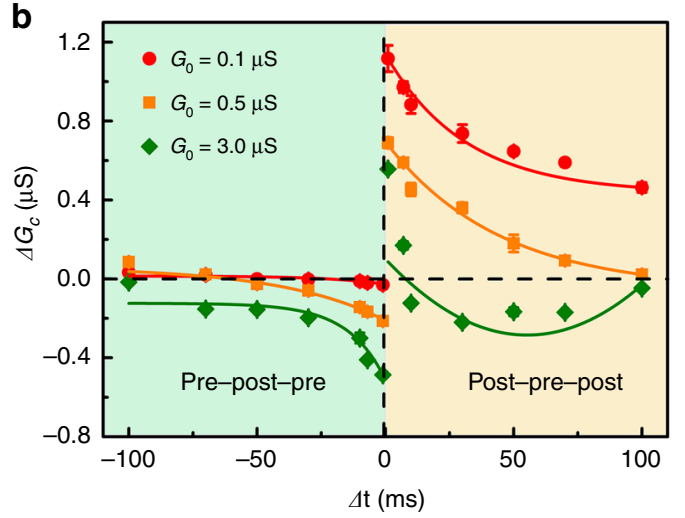

d

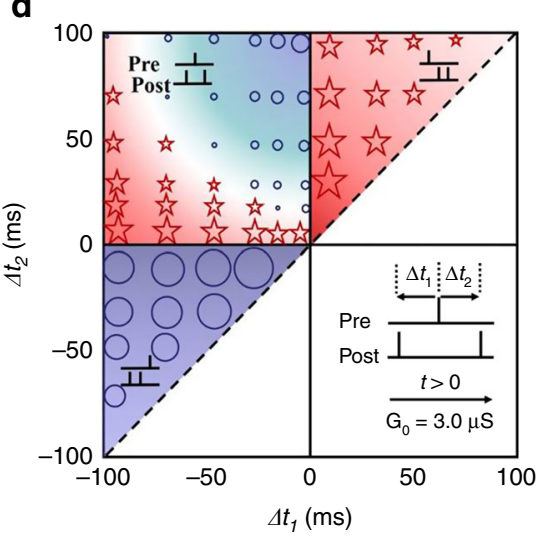

f

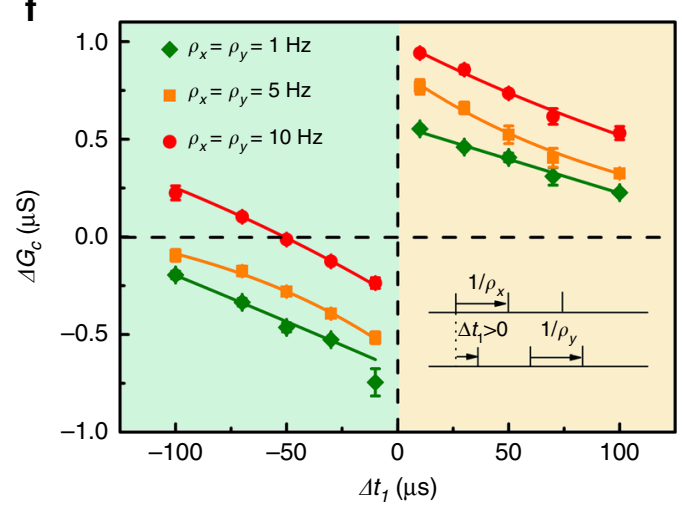

c

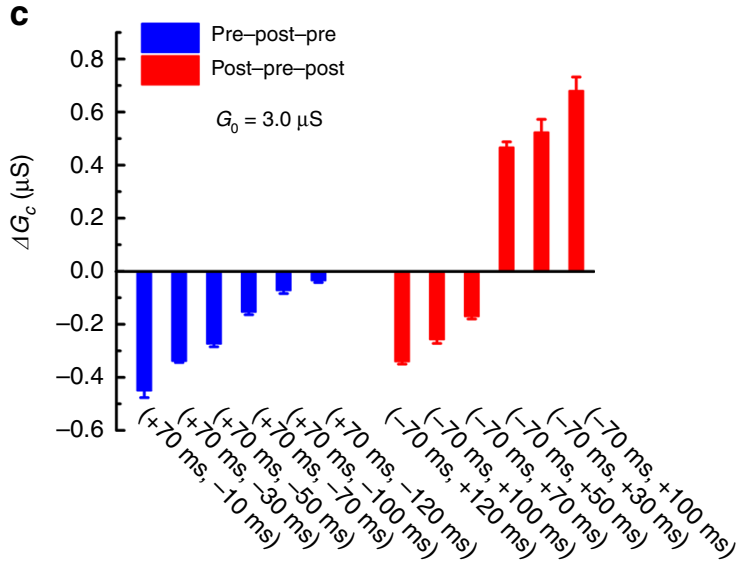

e

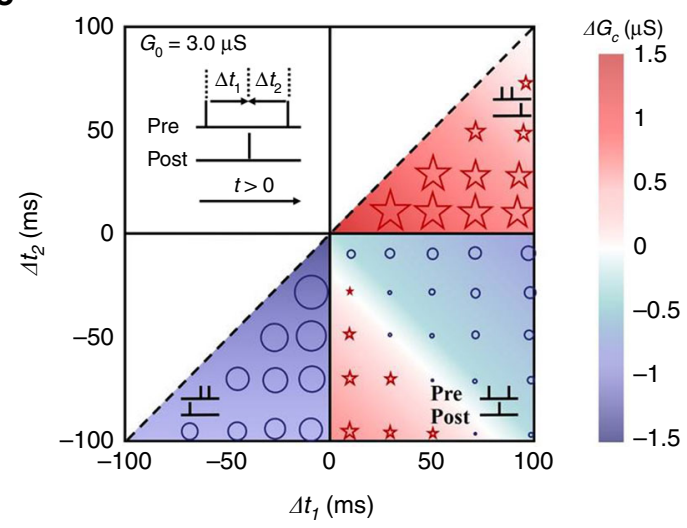

g

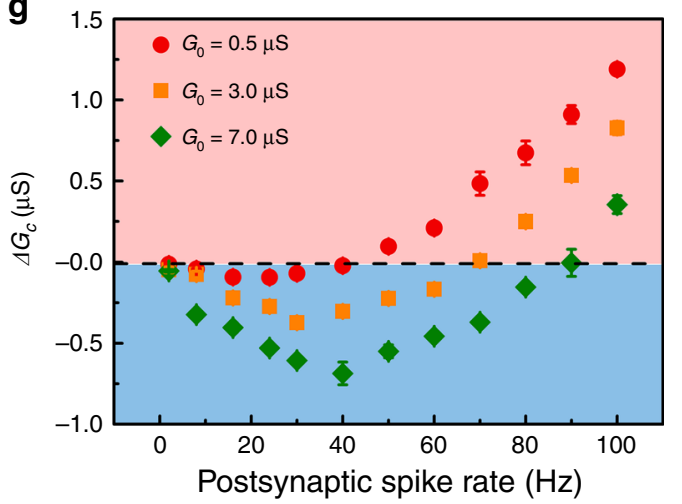

Data indicate that the LTP increases as the spike frequency increasing from $1 \mathrm{~Hz}$ to $10 \mathrm{~Hz}$, while the LTD decreases at increasing the spike frequency. Such dependence on both the presynaptic and postsynaptic spike frequency is consistent with the biological triplet-STDP while it fails to appear in pairedSTDP29,39-42. on both the presynaptic spike rate $\rho_{x}=1 / \Delta t_{r}$ and postsynaptic spike rate $\rho_{y}=1 / \Delta t_{o}$ can be expected, which is also an essential feature in triplet-STDP $29,39-42$. The dependence of LTP/LTD on both the presynaptic and postsynaptic spike frequency was experimentally measured, as shown in Fig. 3f. The $\rho_{x}$ and $\rho_{y}$ share the same value for the spike frequency in this measurement. 
Fig. 3 Demonstration of triplet-STDP and its related $\mathbf{B C M}$ learning rules in $\mathbf{P t} / \mathbf{W O}_{\mathbf{3}-\mathbf{x}} / \mathbf{W}$ memristors. a Schematic of the typical 'post-pre-post' and 'pre-post-pre' triplets, which can be simplified as a superposition of the LTP and LTD processes. Each pre- or postsynaptic spike comprises two pulses with amplitude $\mathrm{V}^{+} / \mathrm{V}^{-}=2 \mathrm{~V} /-2 \mathrm{~V}$ and duration $50 \mathrm{~ms}$. Taking the former as an example, the LTD process activated in the first 'post-pre' pair with spike timing of $\Delta t_{1}<0$ is followed by the LTP process induced by the second 'pre-post' pair with spike timing of $\Delta t_{2}>0$. $\mathbf{b}$ Synaptic modification of triplet-STDP in the 'post-pre-post' and 'pre-post-pre' sequences using symmetrical spike timing $\left|\Delta t_{1}\right|=\left|\Delta t_{2}\right|$, with three levels of the initial $G_{0}$ considered as the learning experiences (i.e., $0.1,0.5$, and $3.0 \mu \mathrm{S}$ ). c Triplet-STDP with asymmetrical spike timing. Red column: $\Delta t_{1}=-70 \mathrm{~ms}, \Delta t_{2}$ is from 10 to $120 \mathrm{~ms}$ in the 'post-prepost' sequence; Blue column: $\Delta t_{1}=+70 \mathrm{~ms}, \Delta t_{2}$ is from -10 to $-120 \mathrm{~ms}$ in the 'pre-post-pre' sequence. $\mathbf{d}$, e Summaries of triplet-STDP results in our experiments, where potentiation or depression with different synaptic weights is obtained using different spike sequences and different timing intervals. The insets show the schematic of 'post-pre-post' and 'pre-post-pre' sequences. Here, both the size of symbols and the background color indicate the magnitude of $\Delta G_{c}$. A relatively high $G_{0}$ of $3.0 \mu \mathrm{S}$ was adopted for the measurements of Fig. 3 (d, e) to highlight the history-dependent characteristics. $\mathbf{f}$ The dependence of $\Delta G_{c}$ on both the presynaptic spike rate $\rho_{x}$ and postsynaptic spike rate $\rho_{y}$. The schematic of the operation signal is shown in the inset, in which three pairs of presynaptic spike and postsynaptic spikes were used. $\mathbf{g}$ Triplet-STDP-based BCM learning rules with the EDE in the low-frequency region and the threshold sliding effect, which is highly similar to the biological BCM curve.

Figure 3d, e can guide us in designing a rational triplet-STDP scheme (e.g., choosing a proper relationship between $\Delta t_{1}$ and $\Delta t_{2}$ ) to fully implement the BCM learning rule. A typical example is shown in Fig. 3g, where the diagonal line of quadrant II (i.e., the 'post-pre-post' triplet with $\left.\left|\Delta t_{1}\right|=\left|\Delta t_{2}\right|\right)$ is chosen, the devices with high $\mathrm{G}_{0}$ (i.e., $0.5,3.0$, and $7.0 \mu \mathrm{S}$ ) were used, and the spike timing sum of $\left|\Delta t_{1}\right|$ and $\left|\Delta t_{2}\right|$ was treated as the frequency of postsynaptic spikes using $\rho_{y}=1 /\left(\left|\Delta t_{1}\right|+\left|\Delta t_{2}\right|\right)$. Figure 3f clearly shows the transition of $\Delta G_{c}$ from depression to potentiation with the spike rate increasing to a threshold value. In particular, the EDE region in the BCM, which was typically absent in previous implementations using the common rate-based postsynaptic spikes $^{34-36}$, was demonstrated for our memristive synapse. Furthermore, the threshold sliding effect with different learning experiences is shown in Fig. $3 \mathrm{~g}$, in which the increase in $G_{0}$ from 0.5 to $7.0 \mu \mathrm{S}$ resulted in a rise of the threshold frequency from 40 to $90 \mathrm{~Hz}$. The forgetting effect thus strengthened with learning experience $G_{0}$ increasing to larger values. Our results are therefore in good agreement with the BCM curve of Fig. 2f, thereby indicating the close reproduction of the BCM learning rule. In fact, if using asymmetrical spike timing with a fixed difference (e.g., $\left|\Delta t_{1}\right|-\left|\Delta t_{2}\right|=20 \mathrm{~ms}$ ) in quadrant II, the tripletSTDP-based BCM rule can also be demonstrated (Supplementary Fig. 5). It is believed that the aforementioned triplet-STDP methods can also be used to obtain the generalized BCM learning rule in other second-order memristors. It is necessary to mention that there are still certain differences between the memristive implementation and the real biological BCM rule. For instance, in the biological BCM rule there is no synaptic weight change for $\rho_{y}=0$ and for any value of $\rho_{x}$, which is instead not the case in our memristive implementation (Fig. $3 \mathrm{~g}$ ). This is mainly because the equivalent pre-/postsynaptic pulses were designed to be equal to simplify the circuit operation, which may be different from the real biological synapse. Anyway, the realization of generalized triplet-STDP-based BCM rule with a long-term nature in memristors has potential applications in high-order spatiotemporal pattern recognition.

Simulation of spatiotemporal selectivity. The realization of high-order spatiotemporal functions in memristive devices is a critical step in building a neuromorphic system that mimics the biological brain. Sun et al. and Wang et al. demonstrated the localization of sound by establishing correlations between the sound azimuth and spike timing ${ }^{54,55}$. Spatiotemporal selectivity (e.g., rate-based orientation selectivity) is another type of classical spatiotemporal function in the visual cortex; however, it has not yet been addressed in memristors. According to the studies of Pfister et al. ${ }^{39,40}$, rate-based orientation selectivity can be expected, following the results of long-term triplet-STDP-based BCM learning rules. A mathematical simulation of the experimental data of the BCM rule is necessary to build the neural network for spatiotemporal selectivity. The characteristics of $\rho_{x^{-}}$and $\rho_{y^{-}}$ dependence (Fig. 3f, g) provided the experimental basis for relating triplet-STDP to the BCM learning rule. The mathematical model of BCM learning can be expressed by refs. $23,39,40,56,57$ :

$$
d G_{c} / d t=\rho_{x} \varphi\left(\rho_{y}, \theta\right)
$$

where $\rho_{x}, \rho_{y}$ are the rates of presynaptic spikes and postsynaptic spikes, respectively and $\theta$ is the threshold rate. Depression of the synaptic weight $\left(\Delta G_{c}<0\right)$ occurs if $\varphi\left(\rho_{y}<\theta, \theta\right)<0$, whereas potentiation of the synaptic weight $\left(\Delta G_{c}>0\right)$ occurs if $\varphi\left(\rho_{y}>\theta, \theta\right)$ $>0$. Finally. there is no synaptic change for $\varphi(0, \theta)=0$. Furthermore, by relating triplet-STDP to the $\mathrm{BCM}$ rule, the change in synaptic weight can be expressed as $39,40,56,57$

$$
d G_{c} / d t=-A_{2}^{-} \tau_{-} \rho_{x} \rho_{y}-A_{3}^{-} \tau_{-} \tau_{x} \rho_{x}^{2} \rho_{y}+A_{2}^{+} \tau_{+} \rho_{x} \rho_{y}+A_{3}^{+} \tau_{+} \tau_{y} \rho_{x} \rho_{y}^{2},
$$

where $A_{2}^{+}$and $A_{2}^{-}$are the amplitude parameters of the paired term contribution for potentiation and depression, where a presynaptic spike triggered before and after a postsynaptic spike can induce LTP and LTD following the classical paired-STDP. The interval time between presynaptic spike and postsynaptic spike is not substantially longer than $\tau_{+}$and $\tau_{-}$. As proposed in the literature $39,40,56$, the presence of a previous postsynaptic spike causes the potentiation contribution of triplet term $A_{3}+$ in addition to the paired term in the 'post-pre-post' triplet. The interval between these two postsynaptic spikes should be in a time window of $\tau_{y}$. Similarly, for the depression contribution of the triplet term, $A_{3}^{-}$and $\tau_{x}$ are the amplitude parameter and interval window of two postsynaptic spikes, respectively. All these parameters were extracted from our experiments on paired and triplet spikes (Supplementary Fig. 6 and Note 3). Following an approach reported in the literature ${ }^{40}$, the minimal triplet rule was considered by setting $A_{2}^{+}=0 \mu \mathrm{S}, A_{2}^{-}=0.02 \mu \mathrm{S}, A_{3}^{+}=0.96 \mu \mathrm{S}, A_{3}{ }^{-}=$ $0 \mu \mathrm{S}, \tau_{+}=38 \mathrm{~ms}, \tau_{-}=30 \mathrm{~ms}, \tau_{x}=16 \mathrm{~ms}$, and $\tau_{y}=0 \mathrm{~ms}$ as parameters in the present study (Supplementary Table 1). Additionally, the sliding threshold effect with different experienced $G_{0}$ is a typical history-dependent property of the BCM learning rule, as mentioned previously. To simulate the sliding threshold effect, threshold $\theta$ differentiating potentiation and depression can be expressed as $39,40,54$

$$
\theta=\left\langle\rho_{\mathrm{y}}^{p}\right\rangle\left(A_{2}^{-} \tau_{-} A_{2}^{+} \tau_{+}\right) /\left(\rho_{0}^{p} A_{3}^{+} \tau_{+} \tau_{y}\right),
$$

where coefficient $\rho_{0}$ and index $p$ were set to $10 \mathrm{~Hz}$ and 2, respectively, for the calculations. Following the literature ${ }^{39,40,56}$, the dependence of $A_{2}^{+}$and $A_{2}^{-}$on the mean firing rate $\left\langle\rho_{y}\right\rangle$ was introduced by setting $A_{2}^{+} \rightarrow A_{2}^{+}\left\langle\rho_{y}^{p}\right\rangle / \rho_{0}^{p}$ and $A_{2}^{-} \rightarrow A_{2}^{-}\left\langle\rho_{y} p\right\rangle /$ $\rho_{0}^{p} \cdot \rho_{y}^{p}$ and $\rho_{0} p$ denote the expectation of the $p^{\text {th }}$ power of $\rho_{y}$ 

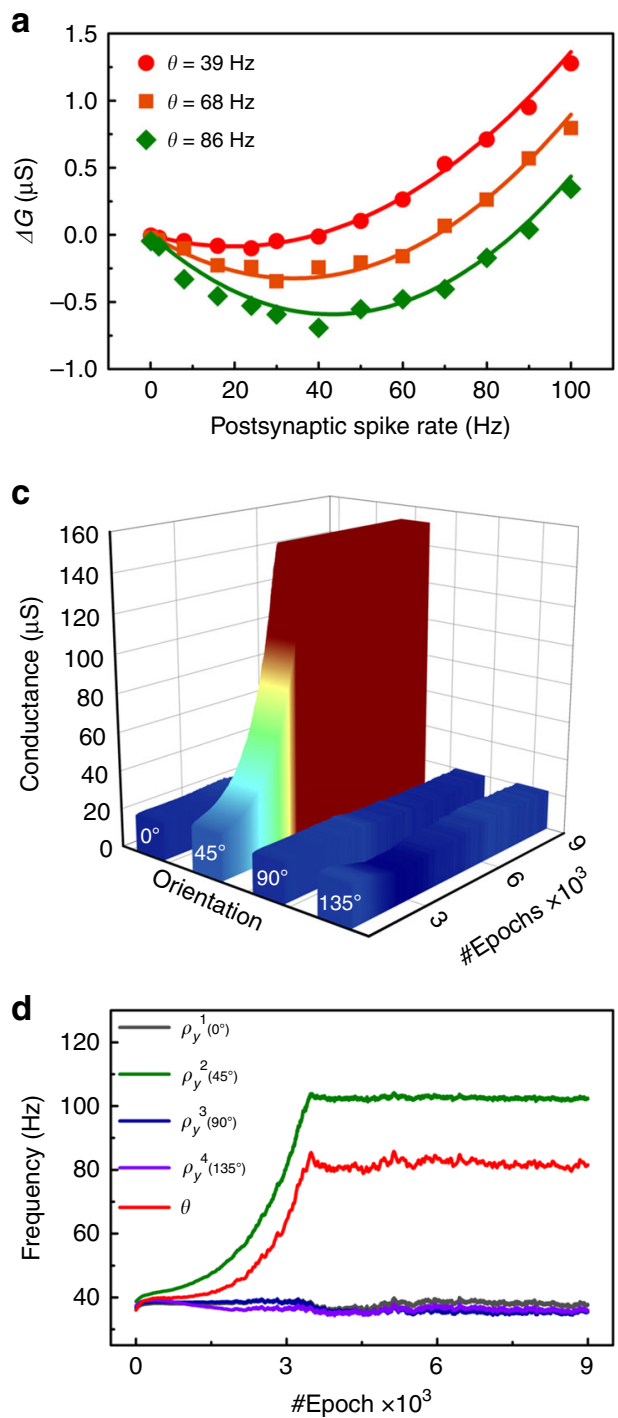

b

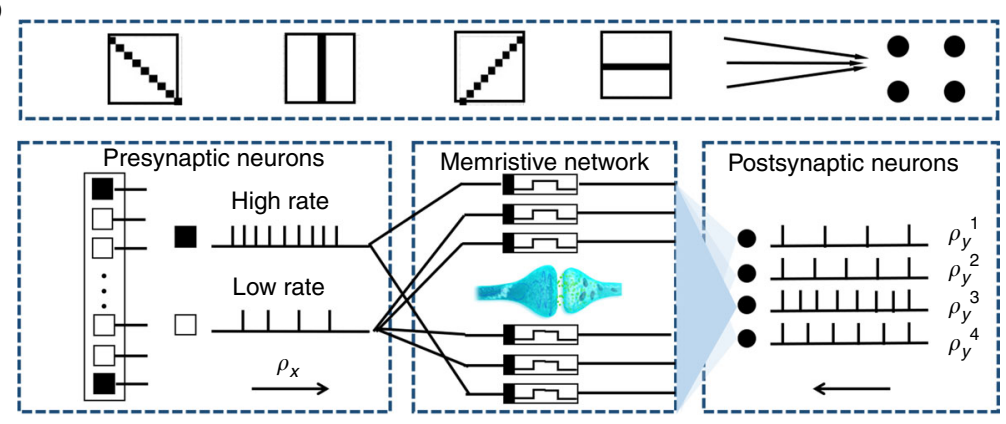

e

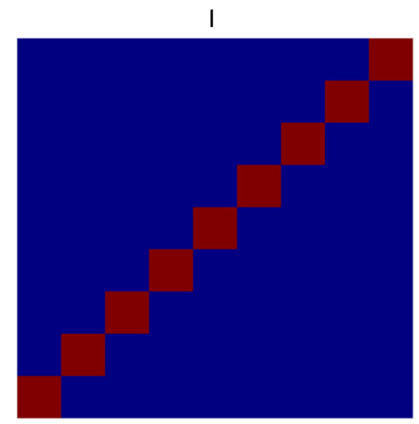

III

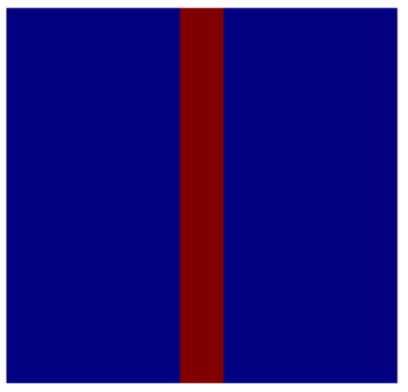

II

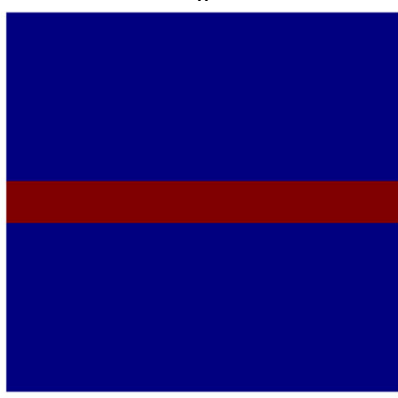

$G(\mu \mathrm{S})$

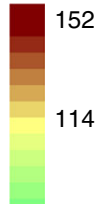

IV

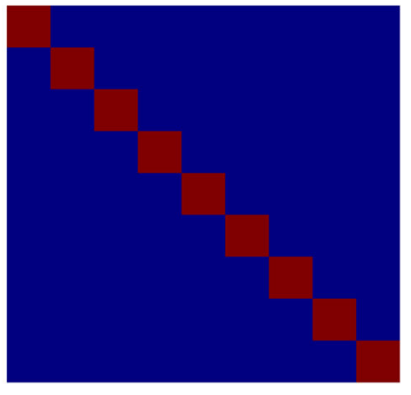

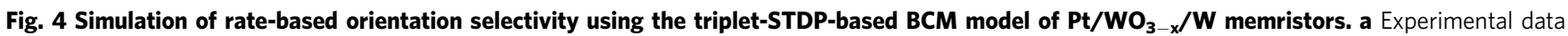
(dots) and calculated curves (lines) of BCM theory, which both have a good fit. b Schematic illustration of the memristor-based feedforward neural network. Presynaptic spikes are generally Poisson spike trains with a mean rate $\left\langle\rho_{x}\right\rangle$. c The $G^{1}$ evolution of the extracted synapses only for the four orientations with the learning epochs. $G^{1}$ denotes the synaptic weight of the synapses connected to the 1st postsynaptic neuron. $\mathbf{d}$ Evolution of $\rho_{y}{ }^{1}$ corresponding to the four orientation bars with the learning epochs. e Color maps of the final synaptic weights in the $9 \times 9$ memristor arrays which reveal the orientation selectivity for four postsynaptic neurons.

and $\rho_{0}$, respectively. For our experimental data, this dependence may be related to the experienced $G_{0}$. As a result, the calculated curve of the BCM theory well fits the experimental data for each $G_{0}$, as illustrated in Fig. 4a, and the threshold sliding effect induced by tuning $G_{0}$ is satisfactorily simulated. The calculated correlations of $\Delta G$ and the postsynaptic spike rate $\rho_{y}$ allow the accurate simulation of neural networks for spatiotemporal patterns.

To extend the triplet-STDP-based BCM model to the application of spatiotemporal patterns, we adopted a two-layer neuromorphic feedforward network, as schematically shown in Fig. 4b. Rate-based orientation selectivity, as the typical input selectivity of spatiotemporal patterns, was numerically simulated using this network following BCM theory. The first layer of presynaptic neurons generate the presynaptic spikes in correspondence of the presentation of a visual pattern, whereas the second layer of postsynaptic neurons generate postsynaptic spikes upon excitation of the first layer. In our network, 81 presynaptic neurons (in a $9 \times 9$ array) and four postsynaptic neurons (with specific number $n=1,2,3,4$ ) were used as the input and output layers, respectively. As a result, there are $81 \times 4=324$ connections (i.e., synapses) between the two layers of the network. Generally, synapses are trained using presynaptic spikes from input neurons with spike rate $\rho_{x}$ and a synchronous spike train from the postsynaptic neuron with rate $\rho_{y}$. At each learning epoch, four patterns, each containing 81 pixels, with different orientation bars (i.e., $0^{\circ}, 45^{\circ}, 90^{\circ}$, and $135^{\circ}$ ) and four random noise patterns were presented to the presynaptic neurons. The presentation of the orientation patterns and noise patterns had equal probability. For the orientation patterns, only the 9 pixels of these four orientation bars had a high spike rate with a Poisson distribution (i.e., $\left\langle\rho_{x}\right\rangle=40 \mathrm{~Hz}$, black), whereas the other 72 pixels had a low spike rate with a Poisson distribution (i.e., $\left\langle\rho_{x}\right\rangle=10$ $\mathrm{Hz}$, white). For the noise inputs, nine pixels were randomly selected with a high spike rate, and the others had a low spike rate. Four $\rho_{y}^{n}$ corresponded to these four postsynaptic neurons. 
Every $\rho_{y}^{n}$ was updated in real time after each pattern or noise according to synaptic weight $G_{m}{ }^{n}$ in the last pattern or noise using the equation $\rho_{y}^{n}=\sum_{m=1}^{81} \rho_{x, m} \times G_{m}^{n}(m=1,2, \ldots 81, n=1$, $2,3,4)^{58,59}$. To achieve the selectivity of multiple orientations, the four $\rho_{y}{ }^{n}$ were compared after each pattern or noise submission. Only the specific postsynaptic neuron with the maximal $\rho_{\mathrm{y}}{ }^{\mathrm{n}}$ could send the fire postsynaptic spikes following the winner-take-all rule, thereby modifying the synaptic weight combined with presynaptic spikes (Supplementary Fig. 7). Correspondingly, the threshold $\theta^{\mathrm{n}}$ was also updated with $\rho_{y}{ }^{n}$ according to Eq. (3).

All the synapses started from a stochastic initial state with low $G_{0}$. Considering the 1st postsynaptic neuron as example, the $G^{1}$ evolution of the extracted synapses only for the four orientations with the learning epoch is shown in Fig. 4c. During the increasing epochs, once a specific orientation was randomly selected by the 1st postsynaptic neuron with strong potentiation, there would be a corresponding higher $\rho_{y}{ }^{1}>\theta^{1}$. Meanwhile, the relatively low $\rho_{y}{ }^{1}$ $<\theta^{1}$ was introduced for the other three orientations, thereby leading to depression. Figure $4 \mathrm{~d}$ shows the $\rho_{y}^{1}$ evolution of the four orientation bars as a function of the learning epochs. From the results of Fig. $4 \mathrm{c}, \mathrm{d}, G^{1}$ and $\rho_{y}{ }^{1}$ of the $45^{\circ}$ orientation bar clearly increase after thousands of learning epochs, which suppressed the values of the other three orientations. Eventually, the orientation of $45^{\circ}$ was selected for the 1st postsynaptic neuron, as shown in Fig. 4e-I, in which the conductance of the nine pixels for the $45^{\circ}$ orientation bar was obviously higher than the others. Simultaneously, although the other three orientations were suppressed in the first postsynaptic neuron, they were selected without supervision by the other three postsynaptic neurons, thereby generating strong potentiation with higher $\rho_{y}{ }^{n}$ $(n=2,3,4)$. As shown in Fig. 4e-II-e-IV, the orientations of $0^{\circ}$, $90^{\circ}$, and $135^{\circ}$ were selected by the 2nd, 3rd, and 4th postsynaptic neurons, respectively, which verifies the feasibility of complete rate-based orientation selectivity in this study. The above results suggest the potential application of the spatiotemporal patterns in our memristors.

\section{Discussion}

We demonstrated a generalized triplet-STDP-based BCM learning rule using a $\mathrm{WO}_{3-\mathrm{x}}$-based second-order memristor. Compared with the BCM rules realized by common rate-based presynaptic spikes, the EDE region missing in previous studies was found in our experimental data. A typical threshold sliding effect that depended on the learning history was also obtained. Furthermore, rate-based orientation selectivity was demonstrated in a feedforward network based on the generalized BCM framework in our memristors by simulation, which indicated its potential feasibility for high-order spatiotemporal patterns. It is noted that there are still certain limitations to a full implementation of the BCM learning at the synaptic level using memristors. For instance, the device physics and signal design may bring differences from the biological synapse, such as the spike-timing region, LTP/LTD window, and specific biological features. Further studies are still required to solve these above limitations toward a fully bio-mimetic BCM rule. It is believed that our study makes a progress towards the biorealistic mimicking of BCM learning rules in memristive synapses and paves the way for the application of memristors to spatiotemporal patterns in the future.

\section{Methods}

Device fabrication. Memristors with a $\mathrm{Pt} / \mathrm{WO}_{3-\mathrm{x}} / \mathrm{W}$ sandwich structure were fabricated on $\mathrm{SiO}_{2} / \mathrm{Si}$ substrates and patterned into a crossbar array with a junction area of $50 \times 50 \mu^{2}$ using a metal mask, as shown in Fig. 1b. Both the W bottom electrode and $\mathrm{WO}_{3-\mathrm{x}}$ film with thicknesses of 100 and $80 \mathrm{~nm}$ were subsequently prepared by RF sputtering using a metal $\mathrm{W}$ target. The $\mathrm{WO}_{3-\mathrm{x}}$ film was grown under a gas pressure of $2 \mathrm{~Pa}\left(\mathrm{Ar}: \mathrm{O}_{2}=3: 1\right)$ at $20{ }^{\circ} \mathrm{C}$. The 80 -nm-thick Pt top electrode was fabricated through electron-beam evaporation.

Electrical measurements. Memristive properties were measured using a self-built test system comprising a sourcemeter (2636A, Keithley), arbitrary function generator (3390, Keithley), oscilloscope (TDS 2012B, Tektronix), and probe station (TTPX, Lake Shore). The positive direction of the bias voltage was defined such that the current flowed from the top to the bottom electrode. To measure the EPSC, the memristor was connected with a load resistor $R_{\text {load }}$ of $1 \mathrm{M} \Omega$ in series, and the voltage drop across the $\mathrm{R}_{\text {load }}$ was monitored by an oscilloscope. Then, the monitored voltage was converted to the current flowing through the memristor. To implement pair- or triplet-STDP, each pre- or postsynaptic spike applied to the top or bottom electrode was composed of a pair of pulses with amplitude $V^{+} / V^{-}=2$ $\mathrm{V} /-2 \mathrm{~V}$ and a width of $50 \mathrm{~ms}$. The initial and final conductance states of the device $\left(G_{i}\right.$ and $\left.G_{\text {final }}\right)$ were readout using a small pulse $[0.2 \mathrm{~V}, 50 \mathrm{~ms}]$ before and after applying the programmable pulses, and the conductance change was defined as $\Delta G_{c}=G_{\text {final }}-G_{\mathrm{i}}$. For the experienced $G_{0}, \Delta G_{c}=G_{\text {final }}-G_{0}$. Both the writing and reading of the memristor were performed in pulse mode.

\section{Data availability}

The data that support the plots within this paper and other findings of this study are available from the corresponding author upon reasonable request.

Received: 31 July 2019; Accepted: 12 February 2020;

Published online: 20 March 2020

\section{References}

1. Mead, C. Neuromorphic electronic systems. P. IEEE 78, 1629-1636 (1990).

2. $\mathrm{Yu}, \mathrm{S}$. et al. A low energy oxide-based electronic synaptic device for neuromorphic visual systems with tolerance to device variation. Adv. Mater. 25, 1774-1779 (2013).

3. Zidan, M. A., Strachan, J. P. \& Lu, W. D. The future of electronics based on memristive systems. Nat. Electron. 1, 22 (2018).

4. Lv, Z., Zhou, Y., Han, S.-T. \& Roy, V. From biomaterial-based data storage to bio-inspired artificial synapse. Mater. Today 21, 537-552 (2018).

5. Zhou, F. et al. Low-voltage, optoelectronic $\mathrm{CH}_{3} \mathrm{NH}_{3} \mathrm{PbI}_{3-\mathrm{x}} \mathrm{Cl}_{\mathrm{x}}$ memory with integrated sensing and logic operations. Adv. Funct. Mater. 28, 1800080 (2018)

6. Zhao, X. et al. Photocatalytic reduction of graphene oxide- $\mathrm{TiO}_{2}$ nanocomposites for improving resistive-switching memory behaviors. Small 14, 1801325 (2018).

7. Yao, P. et al. Face classification using electronic synapses. Nat. Commun. 8, 15199 (2017).

8. Merolla, P. A. et al. A million spiking-neuron integrated circuit with a scalable communication network and interface. Science 345, 668-673 (2014).

9. Wang, Y. et al. Photonic synapses based on inorganic perovskite quantum dots for neuromorphic computing. Adv. Mater. 30, 1802883 (2018).

10. Wang, J. \& Zhuge, F. Memristive synapses for brain-inspired computing. Adv. Mater. Technol. 4, 1800544 (2019).

11. Lin, Y. et al. Analog-digital hybrid memristive devices for image pattern recognition with tunable learning accuracy and speed. Small Methods 3, 1900160 (2019).

12. $\mathrm{Wu}, \mathrm{W}$. et al. A methodology to improve linearity of analog RRAM for neuromorphic computing. in IEEE symposium on VLSI technology 103-104 (IEEE, 2018).

13. Ren, Y. et al. Analytical modeling of organic-inorganic $\mathrm{CH}_{3} \mathrm{NH}_{3} \mathrm{PbI}_{3}$ perovskite resistive switching and its application for neuromorphic recognition. Adv. Theory Simul. 1, 1700035 (2018).

14. Jo, S. H. et al. Nanoscale memristor device as synapse in neuromorphic systems. Nano Lett. 10, 1297-1301 (2010).

15. Wang, Z. et al. Memristors with diffusive dynamics as synaptic emulators for neuromorphic computing. Nat. Mater. 16, 101 (2017).

16. Kim, S. et al. Experimental demonstration of a second-order memristor and its ability to biorealistically implement synaptic plasticity. Nano Lett. 15, 2203-2211 (2015)

17. Pershin, Y. V. \& Di, V. M. Neuromorphic, digital, and quantum computation with memory circuit elements. P. IEEE 100, 2071-2080 (2012).

18. Yu, S., Wu, Y., Jeyasingh, R., Kuzum, D. \& Wong, H.-S. P. An electronic synapse device based on metal oxide resistive switching memory for neuromorphic computation. IEEE T. Electron Dev. 58, 2729-2737 (2011).

19. Zhu, X. et al. Observation of conductance quantization in oxide-based resistive switching memory. Adv. Mater. 24, 3941-3946 (2012).

20. Chang, T., Jo, S.-H. \& Lu, W. Short-term memory to long-term memory transition in a nanoscale memristor. ACS Nano 5, 7669-7676 (2011). 
21. Wang, Z. Q. et al. Synaptic learning and memory functions achieved using oxygen ion migration/diffusion in an amorphous InGaZnO memristor. Adv. Funct. Mater. 22, 2759-2765 (2012).

22. Lin, Y. et al. Transferable and flexible artificial memristive synapse based on $\mathrm{WO}_{\mathrm{x}}$ schottky junction on arbitrary substrates. Adv. Electron. Mater. 4, 1800373 (2018).

23. Ascoli, A., Corinto, F. \& Tetzlaff, R. Generalized boundary condition memristor model. Int. J. Circuit Theory Appl. 44, 60-84 (2016).

24. Wang, Z., Ambrogio, S., Balatti, S. \& Ielmini, D. A 2-transistor/1-resistor artificial synapse capable of communication and stochastic learning in neuromorphic systems. Front. Neurosci. 8, 438 (2015).

25. Tan, Z. H. et al. Synaptic metaplasticity realized in oxide memristive devices. Adv. Mater. 28, 377-384 (2016).

26. Kim, M.-K. \& Lee, J.-S. Short-term plasticity and long-term potentiation in artificial biosynapses with diffusive dynamics. ACS Nano 12, 1680-1687 (2018).

27. Li, B. et al. Mediating short-term plasticity in an Artificial memristive synapse by the orientation of silica mesopores. Adv. Mater. 30, 1706395 (2018).

28. De La Rocha, J., Doiron, B., Shea-Brown, E., Josić, K. \& Reyes, A. Correlation between neural spike trains increases with firing rate. Nature 448, 802 (2007).

29. Sjöström, P. J., Turrigiano, G. G. \& Nelson, S. B. Rate, timing, and cooperativity jointly determine cortical synaptic plasticity. Neuron 32, 1149-1164 (2001).

30. Kirkwood, A., Rioult, M. G. \& Bear, M. F. Experience-dependent modification of synaptic plasticity in visual cortex. Nature 381, 526 (1996).

31. Bear, M. F., Cooper, L. N. \& Ebner, F. F. A physiological basis for a theory of synapse modification. Science 237, 42-48 (1987).

32. Cooper, L. N. \& Bear, M. F. The BCM theory of synapse modification at 30: interaction of theory with experiment. Nat. Rev. Neurosci. 13, 798 (2012).

33. Zenke, F., Agnes, E. J. \& Gerstner, W. Diverse synaptic plasticity mechanisms orchestrated to form and retrieve memories in spiking neural networks. Nat. Commun. 6, 6922 (2015).

34. Du, C., Ma, W., Chang, T., Sheridan, P. \& Lu, W. D. Biorealistic implementation of synaptic functions with oxide memristors through internal ionic dynamics. Adv. Funct. Mater. 25, 4290-4299 (2015).

35. Yin, J. et al. Adaptive crystallite kinetics in homogenous bilayer oxide memristor for emulating diverse synaptic plasticity. Adv. Funct. Mater. 28, 1706927 (2018).

36. Xiong, J. et al. Bienenstock, Cooper, and Munro Learning Rules Realized in Second-order memristors with tunable forgetting rate. Adv. Funct. Mater. 29, 1807316 (2019).

37. Kurashige, H. \& Sakai, Y. BCM-type synaptic plasticity model using a linear summation of calcium elevations as a sliding threshold. in International Conference on Neural Information Processing 19-29 (Springer, 2006).

38. Artola, A. \& Singer, W. Long-term depression of excitatory synaptic transmission and its relationship to long-term potentiation. Trends Neurosci. 16, 480-487 (1993).

39. Gjorgjieva, J., Clopath, C., Audet, J. \& Pfister, J.-P. A triplet spiketiming-dependent plasticity model generalizes the Bienenstock-CooperMunro rule to higher-order spatiotemporal correlations. Pro. Natl Acad. Sci. USA 108, 19383-19388 (2011).

40. Pfister, J.-P. \& Gerstner, W. Triplets of spikes in a model of spike timingdependent plasticity. J. Neurosci. 26, 9673-9682 (2006).

41. Froemke, R. C. \& Dan, Y. Spike-timing-dependent synaptic modification induced by natural spike trains. Nature 416, 433 (2002).

42. Wang, H.-X., Gerkin, R. C., Nauen, D. W. \& Bi, G.-Q. Coactivation and timing-dependent integration of synaptic potentiation and depression. Nat. Neurosci. 8, 187 (2005).

43. Ahmed, T. et al. High order synaptic learning in neuro-mimicking resistive memories. arXiv preprint arXiv, 180406688 (2018).

44. Yang, R. et al. Synaptic suppression triplet-STDP learning rule realized in second-order memristors. Adv. Funct. Mater. 28, 1704455 (2018).

45. Milo, V. et al. A 4-transistors/one-resistor hybrid synapse based on resistive switching memory (RRAM) capable of spike-rate dependent plasticity (SRDP). IEEE Trans. VLSI 26, 2806-2815 (2018).

46. Wang, W. et al. Surface diffusion-limited lifetime of silver and copper nanofilaments in resistive switching devices. Nat. Commun. 10, 81 (2019).

47. Abbott, L. F. \& Nelson, S. B. Synaptic plasticity: taming the beast. Nat. Neurosci. 3, 1178 (2000).

48. Baeumer, C. et al. Verification of redox-processes as switching and retention failure mechanisms in $\mathrm{Nb}: \mathrm{SrTiO}_{3} /$ metal devices. Nanoscale 8, 13967-13975 (2016).

49. Yang, R. et al. Synaptic plasticity and memory functions achieved in a $\mathrm{WO}_{3-\mathrm{x}^{-}}$ based nanoionics device by using the principle of atomic switch operation. Nanotechnology 24, 384003 (2013).
50. Yang, C. S. et al. All-solid-state synaptic transistor with ultralow conductance for neuromorphic computing. Adv. Funct. Mater. 28, 1804170 (2018).

51. Turrigiano, G. G. \& Nelson, S. B. Homeostatic plasticity in the developing nervous system. Nat. Rev. Neurosci. 5, 97 (2004).

52. Rachmuth, G., Shouval, H. Z., Bear, M. F. \& Poon, C.-S. A biophysically-based neuromorphic model of spike rate-and timing-dependent plasticity. Pro. Natl Acad. Sci. USA 108, E1266-E1274 (2011)

53. Burkitt, A. N., Meffin, H. \& Grayden, D. B. Spike-timing-dependent plasticity: the relationship to rate-based learning for models with weight dynamics determined by a stable fixed point. Neural Comput. 16, 885-940 (2004).

54. Sun, L. et al. Synaptic computation enabled by joule heating of single-layered semiconductors for sound localization. Nano Lett. 18, 3229-3234 (2018)

55. Wang, W. et al. Learning of spatiotemporal patterns in a spiking neural network with resistive switching synapses. Sci. Adv. 4, eaat4752 (2018).

56. Azghadi, M. R., Al-Sarawi, S., Iannella, N. \& Abbott, D. Design and implementation of BCM rule based on spike-timing dependent plasticity. in Neural Networks (IJCNN), International Joint Conference on 1-7 (IEEE, 2012).

57. Cai, W., Ellinger, F. \& Tetzlaff, R. Neuronal synapse as a memristor: modeling pair-and triplet-based STDP rule. IEEE Trans. Biomed. Circuits Syst. 9, 87-95 (2014).

58. Lawlor, M. \& Zucker, S. W. Feedforward learning of mixture models. Adv. Neural Inf. Process. Syst. 3, 2564-2572 (2014).

59. Udeigwe, L. C., Munro, P. W. \& Ermentrout, G. B. Emergent dynamical properties of the BCM learning rule. J. Math. Neurosci. 7, 2 (2017).

\section{Acknowledgements}

This work was supported by the NSFC Program (Nos. 51701037, 51732003, 51872043, 61774031, 11974072, and 61574031), the "111" Project (No. B13013), the fund from Ministry of education (614A02033414), the fund from MOST (No. 2018YFE0118300, 2019YFB2205100) and the European Research Council (ERC) under the European Union's Horizon 2020 research and innovation programme (grant agreement No. 648635). D.I. acknowledges support from the Ministero degli Affari Esteri e della Cooperazione Internazionale (grant number PGR01011).

\section{Author contributions}

Z.W. and T.Z. contributed equally to this work. Z.W, H.X., YC.L, and D.I. conceived the original concept and initiated the work. T.Z. fabricated devices. T.Z., Y.L., and X.Z. performed measurements. Y.R. performed simulations. H.X., D.I., and YC.L. contributed to analysis of the data and discussion. Z.W. wrote the manuscript. All authors commented on the manuscript.

\section{Competing interests}

The authors declare no competing interests.

\section{Additional information}

Supplementary information is available for this paper at https://doi.org/10.1038/s41467020-15158-3.

Correspondence and requests for materials should be addressed to H.X., Y.L. or D.I

Peer review information Nature Communications thanks Alon Ascoli and the other anonymous, reviewer(s) for their contribution to the peer review of this work.

Reprints and permission information is available at http://www.nature.com/reprints

Publisher's note Springer Nature remains neutral with regard to jurisdictional claims in published maps and institutional affiliations.

Open Access This article is licensed under a Creative Commons Attribution 4.0 International License, which permits use, sharing, adaptation, distribution and reproduction in any medium or format, as long as you give appropriate credit to the original author(s) and the source, provide a link to the Creative Commons license, and indicate if changes were made. The images or other third party material in this article are included in the article's Creative Commons license, unless indicated otherwise in a credit line to the material. If material is not included in the article's Creative Commons license and your intended use is not permitted by statutory regulation or exceeds the permitted use, you will need to obtain permission directly from the copyright holder. To view a copy of this license, visit http://creativecommons.org/ licenses/by/4.0/

(c) The Author(s) 2020 